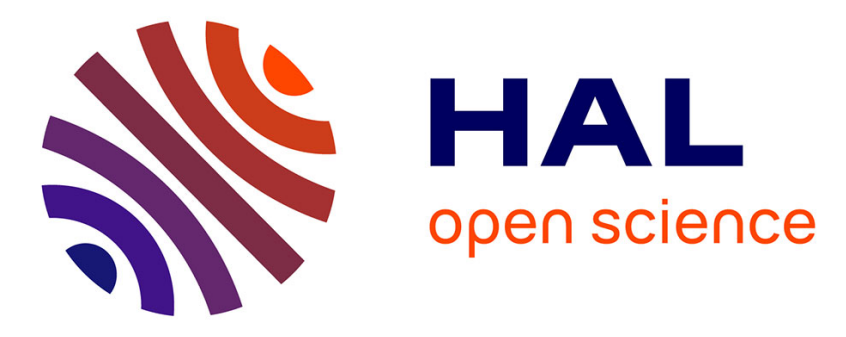

\title{
Walking gait step length asymmetry induced by handheld device
}

Mahdi Abid, Valérie Renaudin, Yannick Aoustin, Eric Le Carpentier, Thomas Robert

\section{- To cite this version:}

Mahdi Abid, Valérie Renaudin, Yannick Aoustin, Eric Le Carpentier, Thomas Robert. Walking gait step length asymmetry induced by handheld device. IEEE Transactions on Neural Systems and Rehabilitation Engineering, 2017, 25 (11), pp.2075-2083. 10.1109/TNSRE.2017.2705285 . hal01623521v2

\section{HAL Id: hal-01623521 \\ https://hal.science/hal-01623521v2}

Submitted on 6 Mar 2018

HAL is a multi-disciplinary open access archive for the deposit and dissemination of scientific research documents, whether they are published or not. The documents may come from teaching and research institutions in France or abroad, or from public or private research centers.
L'archive ouverte pluridisciplinaire HAL, est destinée au dépôt et à la diffusion de documents scientifiques de niveau recherche, publiés ou non, émanant des établissements d'enseignement et de recherche français ou étrangers, des laboratoires publics ou privés. 


\title{
Walking gait step length asymmetry induced by handheld device
}

\author{
Mahdi Abid, Valerie Renaudin*, Yannick Aoustin, Eric Le-Carpentier, and Thomas Robert
}

\begin{abstract}
The modeling and feature extraction of human gait motion are crucial in biomechanics studies, human localization and robotics applications. Recent studies in pedestrian navigation aim at extracting gait features based on the data of low-cost sensors embedded in handheld devices such as smartphones. The general assumption in Pedestrian Dead Reckoning (PDR) strategy for navigation application is that the presence of a device in hand does not impact the gait symmetry and that all steps are identical. This hypothesis, which is used to estimate the traveled distance, is investigated in this paper with an experimental study. Ten healthy volunteers participated in motion lab tests with a $0.190 \mathrm{~kg}$ device in hand. Several walking trials with different device carrying modes and several gait speeds were performed. For a fixed walking speed, it is shown that the steps differ in their duration when holding a mass equivalent to a smartphone mass, which invalidates classical symmetry hypothesis in PDR step length modeling. It is also shown that this hypothesis can lead to a 2.5 to $6.3 \%$ error on the PDR estimated traveled distance for the different walking trials.
\end{abstract}

Index Terms-Handheld devices, Human walking gait, Pedestrian navigation, Step level symmetry.

\section{INTRODUCTION}

$\mathbf{M}$ ORE and more connected objects are invented and commercialized to assist daily life activities. Among the targeted applications are health monitoring, physical activity measuring and navigation aiding. Whereas smartphones, smartwatches or smart-bands are successfully used to count the number of steps in daily routine or record race trajectories using satellites positioning technology, like Global Navigation Satellites Systems (GNSS), their use for navigation remains challenging because of the complexity of both the human movements and the perturbation of radio signals in the traversed surroundings. Globally the development of innovative positioning and navigation algorithms is driven by the fact that travelers are more and more connected to the Internet of Things all along their journey. In this context, it is very interesting to use consumer wrist sensors to provide guidance information and traffic data but existing positioning and navigation technologies fail to address this expectation.

Manuscript submitted xx xx, 2016; This work was supported by Région des Pays de la Loire, LMA Project (Longévité, Mobilité and Autonomie) des Pays de la Loire and the Marie Curie Actions fellowships for the project smartWALK. Asterisk indicates corresponding author.

M. Abid and V. Renaudin are with IFSTTAR, GEOLOC Laboratory, Route de Bouaye CS4, 44344 Bouguenais, France (email: mahdi.abid@ifsttar.fr; valerie.renaudin@ifsttar.fr).

Y. Aoustin and E. Le-Carpentier are with LS2N, UMR 6004, École Centrale de Nantes, Université de Nantes, France (email: Yannick.Aoustin@univnantes.fr; eric.le-carpentier@ec-nantes.fr).

T. Robert is with IFSTTAR, Biomechanics and Impact Mechanics Laboratory (LBMC), Bron, France (email: thomas.robert@ifsttar.fr).

Manuscript received XX XX, 2016; XX.
One difficulty is that these objects are attached to the upper parts of the body whereas the human gait is mainly driven by the lower parts of the body. The choice of the measuring point has a strong impact on the performances of the activity classification process. Globally wrist worn sensors give lower performances for analyzing human gait than ear, chest or foot sensors, which correspond to relatively fixed positions during the displacement [1]. A reason is that wrist sensors are far away from the legs and they measure many movements that are not related to the global locomotion. Indeed, upper body movements can be of very high amplitude and may cover the motion driven by the legs. To overcome this challenge, existing personal navigation solutions based on wrist sensors monitor human motions and model human gait in order to derive pedestrians' tracks [2]-[4].

GNSS are the prime technologies for navigation. Trajectories are derived by differentiating GNSS point coordinates and comparing them with pre-processed itineraries to provide guidance data. Pedestrians' positions are computed by intersecting lines that are derived from times of flight measured on the signals broadcasted by the GNSS satellites. A minimum of four satellites must be seen in direct line of sight to estimate the coordinates [5]. If the satellites signals are reflected by surrounding obstacles, the distances are over estimated introducing errors in the location estimate. As a consequence, the accuracy, the availability and the continuity of GNSS positioning cannot be guaranteed in indoor spaces and urban canyons where the signals are strongly attenuated and affected by multipath. Because humans spend more and more time indoors [6], alternative solutions are sought.

One solution consists in fusing natural signals such as accelerations, angular rates or magnetic field sensed by an inertial and magnetic mobile unit (MIMU) [7] with GNSS data. One advantage of this approach is that these signals rely on external forces that are always available in all environments (indoors, outdoors). MIMUs and GNSS receivers are often embedded in unobtrusive portable devices, carried in hands or kept in bags. Two signal processing methods exist for location estimation: the strap-down mechanization [8] and the Pedestrian Dead Reckoning technique [9]. Strap down mechanisation double integrates the accelerations to estimate the travel distance whereas PDR uses step length models. Both methods provide good results when the sensors are attached to some relatively stable parts of human body (e.g. belt or foot). With foot mounted sensors, zero velocity phases of the walking gait can be sensed and used to calibrate the sensor errors that are inherent to their low cost nature [10]. With wrist mounted sensors, these phases do not exist and 


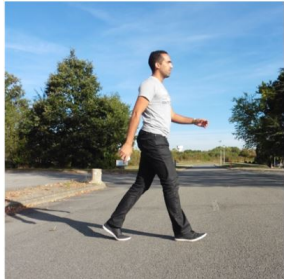

SWINGING

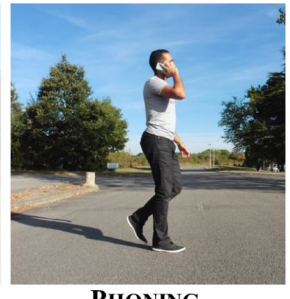

PHONING

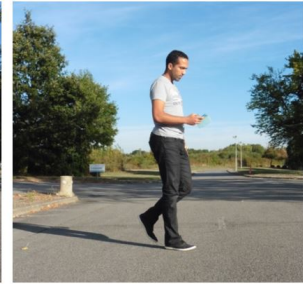

TEXTING
Fig. 1: Different carrying modes of the handheld device

the PDR strategy must be adopted. It consists in estimating step lengths and walking directions to calculate the current coordinates based on the previous one. MIMU data are first filtered to recognize the activity completed by the different device carrying modes: static, swinging, texting, phoning, handbag/trouser and irregular motion modes [3] (see Fig. 1). Phoning and texting modes form one class corresponding to relatively stable lever arm between the sensor and the pedestrian center of mass locations. Parametric models based on the height of human participant and the step frequency, estimated using the acceleration [11], [12], are used to estimate the step length [13]. This approach is only partially successful because it relies on simplifications that are based on empirical observations from biomechanics. One common assumption is that the human gait is step symmetric irrespective of the presence of a mass in hand as it is the case with handheld sensors [13]-[15]. This hypothesis is investigated in this paper following an experimental approach.

Because a huge amount of experiments would be necessary to cover the diversity of human gaits, a simulation tool, which can be parameterized to test the impact of different features on the walking gait, is under development. It is the first outcome of this biped based simulator, optimized at the stride level, that a possible impact of holding a small mass in hand on the walking gait symmetry was first observed. This preliminary human gait modeling over a stride along with the step length estimated with varying walking speeds are presented in section II. To further investigate the impact of a mass in hand, experimental tests were conducted in a motion laboratory with ten persons walking on a treadmill holding a $0.190 \mathrm{~kg}$ sensor in hand in different carrying modes. They are presented in section III and analyzed using biomechanics based statistical approach in section IV. Section V assesses the error induced by assuming step length symmetric model in PDR algorithms for navigation applications.

\section{HUMAN GAIT MODELING OVER A STRIDE}

In the following, the modeling of cyclic walking gait over a stride based on an enhanced 2D biped model is presented. The aim is to model the human behavior for several walking speeds, for natural walking or walking with a device in hand, and for different user morphologies. This human gait model is also adapted to study the impact of different device carrying modes on gait cycle characteristics.

\section{A. Existing human gait simulation with upper limbs modeling}

Human walking is a complex dynamic activity since the human structure has a high degree of freedom (DOF) and 3D deformable frames [16]. To overcome these problems, many researches on gait pattern generation define the natural gait of the biped by the movement that minimizes its energy [17]-[20] In this context, an important challenge is to better understand the role of upper limbs in human walking since there is an infinity of solutions to produce the same locomotion patterns due to the human motor system actuation redundancy [21].

Using parametric optimization for a $2 \mathrm{D}$ biped gait generation, it was proven that active arm swinging results in a decrease of energetic criterion with respect to passive arm swinging case [22]. This optimization technique is also used in [23] to study different gait scenarios over a step. The model used in this study considered one-link arms. However to study different carrying modes such as texting/phoning modes, two links per arm are at least needed. Moreover, to study the possible impact of upper limbs constraints on the step level symmetry, the extension of optimization to a stride is necessary. Two consecutive steps are considered in the new model as we aim to model the human periodical gait over its cycle which is the stride.

\section{B. Extended 2D biped model}

The proposed biped in the sagittal plane is composed of two identical three-link legs, two identical two-link arms and a head-trunk segment (Fig. 2). All the joints are revolute and supposed to be frictionless and independently actuated. All links are modeled by rigid and massive segments. An active role of arms during locomotion is assumed in this model.

Hands are neglected in this model and the head and the trunk are merged in one link. The handheld device is modeled as a punctual mass object whose inertias are neglected. This mass is rigidly attached to the forearm and their mass and inertial properties are merged. To adjust this $2 \mathrm{D}$ biped to a real human subject, body segment inertial parameters (BSIPs) must be estimated. First, segments lengths are estimated according to the anatomical definition proposed in the anthropometric tables [24], [25]. Then DeLeva regressions are used to estimate the BSIPs [25]. These regressions, whose values were normalized using data from different anthropometric surveys of Caucasian populations, are suitable for 2D studies [25].

\section{Optimization algorithm}

A parametric optimization algorithm using $S Q P$ method (Sequential Quadratic Programming) [27] is developed to solve for the nonlinear constrained problem. The gait cycle is composed of successive single support phases with flat contact between the support foot and the ground and instantaneous Double Support (DS) phases. $3^{\text {rd }}$ order polynomial functions are used to interpolate joint motion histories for each step. The parameters defining the polynomials coefficients are determined using an optimization under several nonlinear constraints on the validity of impact, ground reactions, dynamic equilibrium, joint positions, rates and torques. The 


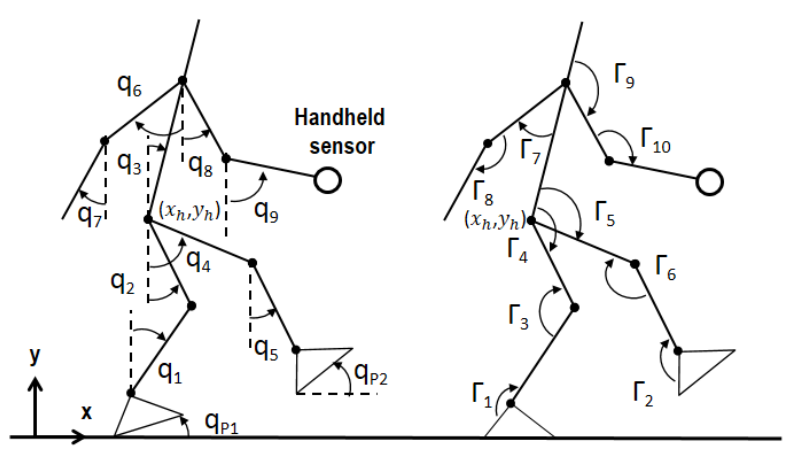

Fig. 2: Schematic in the sagittal plane of the 2D Biped with its different DOF and corresponding actuators [26]

cost function to minimize is defined by the torques calculated using the inverse dynamic model [28]. We consider the integral of torque norm. This algorithm is tested for the biped model fitted to one participant $($ mass $=100 \mathrm{~kg}$, height $=1.85 \mathrm{~m}$ ).

\section{Results}

The numerical simulations carried out for several gait velocities show the following results:

1. During the optimized gait cycle, each arm reciprocally flex and extend with phase opposite to its contralateral leg. The range of arc of motion used at the shoulders is $16.5^{\circ}-31.4^{\circ}$ for walking velocity varying from 0.4 to $1.6 \mathrm{~m} / \mathrm{s}$. These values of optimal arm swing are close to biomechanical data found in [29] (e.g. an average arc of motion of $32^{\circ}$ during moderate gait speed).

2. The effort criterion is higher for the texting mode than in the normal swinging which supports the energetic effect of natural walking reflected in the low cost for arms swinging found in [12].

3. The durations of both steps (right and left) are no longer equal when switching the handheld mass to $0.19 \mathrm{~kg}$. This outcome is illustrated in Fig. 3 where the right/left step durations versus the gait velocity are plotted for texting and swinging conditions.

The last result suggests that during walking with a device in hand, the added mass or the constrained upper limbs configuration may lead to a step level asymmetry of the gait cycle. This observation refutes the hypothesis that all steps are identical, which is commonly considered in PDR navigation solution. To check its validity, this result is further investigated with motion capture experiments that are detailed in section III and analyzed in section IV.

\section{EXPERIMENTAL APPROACH}

The following experiments have been designed to study the influence of a mass carried in hand on the walking gait cycles. It is hypothesized that the presence of handheld mass may alter the step level symmetry of the walking gait. This section starts with the experimental setup and the scenarios. The parameters used to assess the symmetry of the walking gait are then presented followed by the analysis of the experimental data.

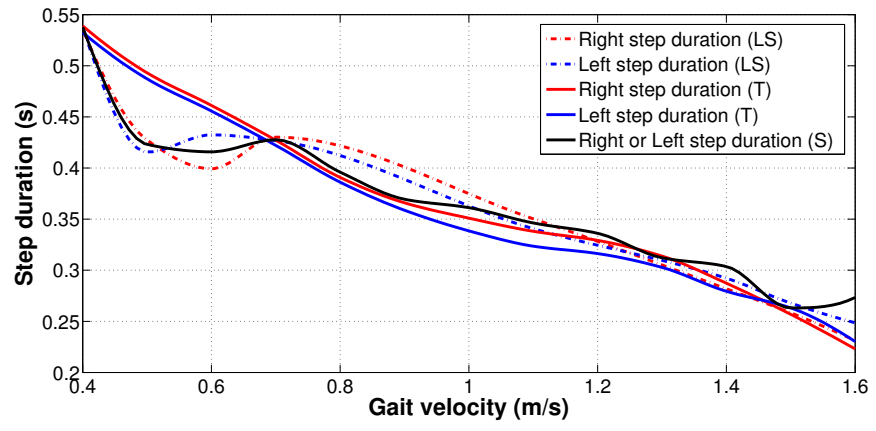

Fig. 3: Right (red) and left (blue) steps durations versus the walking speed for the loaded swinging (LS: dash-dot curves) and texting (T: solid curves) modes. "Loaded" means with the presence of a mass held in hand. The curve in black corresponds to unloaded swinging $(\mathrm{S})$ case.

\section{A. Experimental Setup}

The experiments were conducted in a motion capture room equipped with ART IR tracking system working at a $60 \mathrm{~Hz}$ sampling frequency where a treadmill was installed (Fig. 4). The tracking system consists of eight cameras (four on the floor and four close to the ceiling) and ART MoCap target set, i.e. passive reflective clusters of markers. The handheld device weighed $0.19 \mathrm{~kg}$, which corresponds to the mass of a smartphone (Fig. 5). Optical markers' assemblies were taped to it, to both feet and to the belt for getting accurate reference trajectories of these locations.

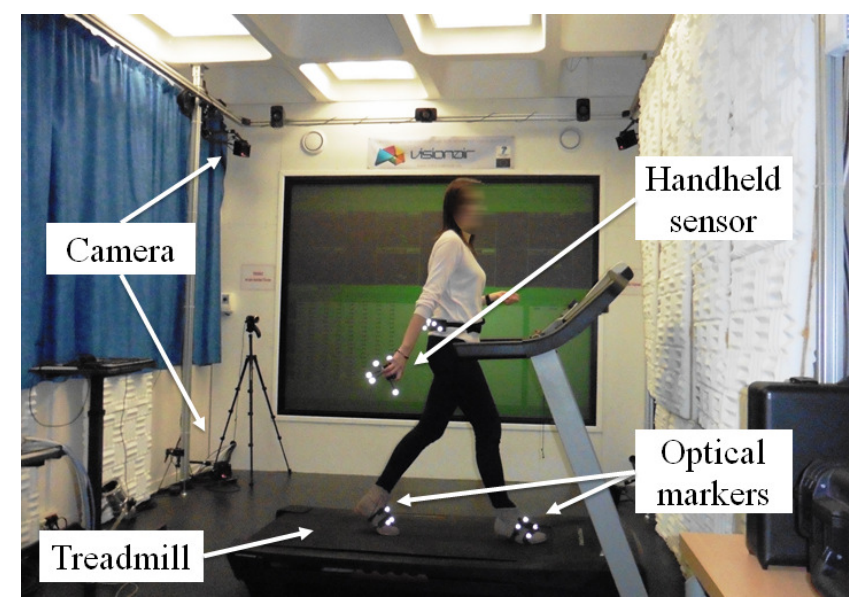

Fig. 4: Experimental setup

Ten healthy individuals, six men and four women, with a 31 year average age (age range 21-52 years), $1.72 \mathrm{~m}$ average height (with shoes) and $74.9 \mathrm{~kg}$ average mass, volunteered to participate in this experimentation. All participants were provided written informed consent (institutional review board ethical process). The trials started after all participants were acquainted with the treadmill and the device at the three following velocities: $V_{1}=3.6 \mathrm{~km} / \mathrm{h}(1 \mathrm{~m} / \mathrm{s}), V_{2}=5 \mathrm{~km} / \mathrm{h}(1.38$ $\mathrm{m} / \mathrm{s})$ and $V_{3}=5.8 \mathrm{~km} / \mathrm{h}(1.61 \mathrm{~m} / \mathrm{s})$. Data record started only after a participant had reached a steady state at each walking 


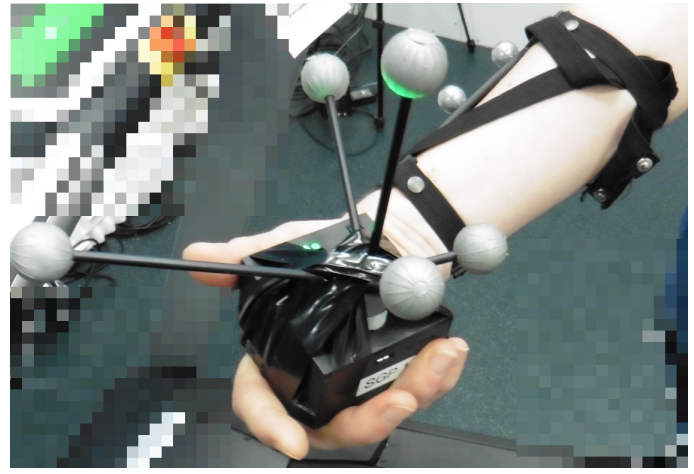

Fig. 5: Handheld device equipped with an optical markers' assembly

speed. In addition, all participants were unaware of the specific data processing.

Walking on a treadmill is acknowledged as being different from natural walking. It is demonstrated that the treadmill induces a more symmetric and consistent gait pattern [30]. The study requires accurate measurements of the markers' positions in order to derive gait features. This was made possible with a qualified motion capture system whose workspace is limited to approximately $10 \mathrm{~m}^{2}$. Natural walking could not be possible in this room because only too short distances (in circles) could be traveled by the individuals in this space. The use of a treadmill was preferred to test straight walking scenarios. Another advantage of this alternative is that the study could be conducted for several steady walking speeds.

The experiments were conceived to test the influence of holding a connected object in hand on the gait cycle. Therefore experiments were conducted with and without the small mass in hand for all participants. Three different device carrying modes, corresponding to different scenarios, were defined for the experiments. They are :

- the unloaded swinging mode: the participant is naturally walking with no mass in hand, i.e. the arms are freely oscilating during the walk;

- the loaded swinging mode: the participant is naturally walking while holding the device in his/her swinging hand;

- the texting mode that corresponds to a body fixed device carrying mode: the participant is instructed to walk watching his/her device's screen so that the right upper limbs configuration is comfortable for reading or texting on the device.

Each scenario was performed for all three testing velocities $V_{1}, V_{2}$ and $V_{3}$.

\section{B. Parameters used to assess the gait symmetry}

Three different features are defined to analyze the symmetry of the walking gait. They are now detailed.

- Step Duration. This is the time elapsed between two successive detected steps. Step durations are calculated for both legs. Labels are used to differentiate right step duration from left step duration.
- Temporal Symmetry Index (TSI step) over step. TSI over step is defined as the ratio of time duration of the step on the device's side over the total duration of a stride as defined in (1). This index is useful for studying the temporal step symmetry. For step symmetric walking gait, TSI over step should be 0.5 .

$$
\mathrm{TSI}_{\text {step }}=\frac{\text { Time duration of step on the device's side }}{\text { Total time duration of the stride }}
$$

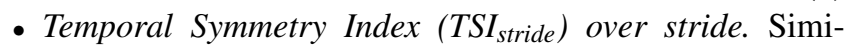
larly, TSI over stride is defined as the ratio of time duration of the first stride over the duration of two successive strides. This index is useful for assessing the step detection method. An accurate step detection should result in a stride level symmetric gait irrespective of the presence of mass in one hand [31].

\section{Experimental Gait features extraction}

Gait cycle is characterized by the occurrence of a stable foot's point during the flat foot phase, which corresponds to step events. The Zero Velocity Detection (ZVD) method has widely been adopted in the navigation field to detect steps. It is based on an acceleration moving variance detector and it searches for the periods when the foot-mounted cluster of markers is stationary [10]. In our study, it is applied to extract the step events from the acceleration data. It is obtained by double differentiating the markers' positions, which are tracked by the motion capture system. In order to remove the noise induced by the derivation, the data are low-pass filtered with a zero-phase forward and reverse second order Butterworth filter with a cut-off frequency of $10 \mathrm{~Hz}$.

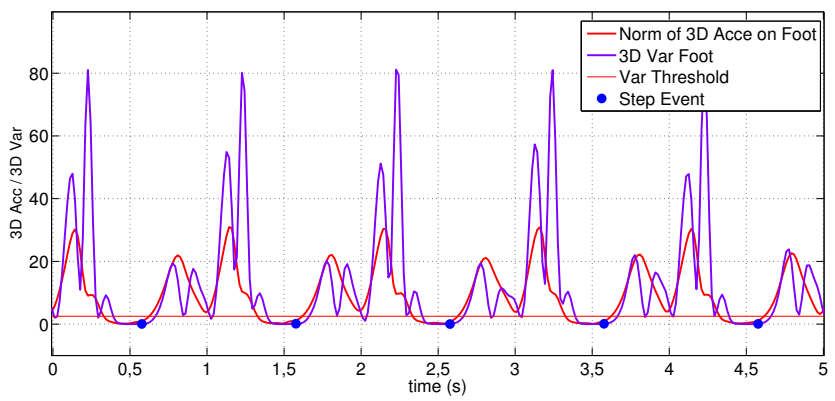

Fig. 6: Step detection using the acceleration-moving variance detector

The variance of the norm of the acceleration vector is calculated over a sliding window and compared to an adaptive threshold. The outcome is illustrated in Fig. 6, where the blue dots correspond to the detected stance phases, i.e. the steps. The detection is performed for both the right and left feet in order to estimate the step durations.

\section{ANALYSIS OF THE EXPERIMENTAL DATA}

200 continuous steps ( 100 continuous strides) were analyzed for each trial using the ZVD method. To assess the accuracy of this step detection method, TSI over strides were analyzed. It 
was found that for all subjects, and across any of the conditions evaluated, the $\mathrm{TSI}_{\text {stride }}$ values follow Gaussian distributions and are centered around 0.5 , with all standard deviations remaining below $6.10^{-3}$. This result shows that the walking gait is stride symmetric irrespective to the presence of mass in one hand. This asserts the hypothesis $\left(H_{1}\right)$ found in biomechanics litterature that is further used to build the model in the simulation. Then, this analysis validates the precision of ZVD method.

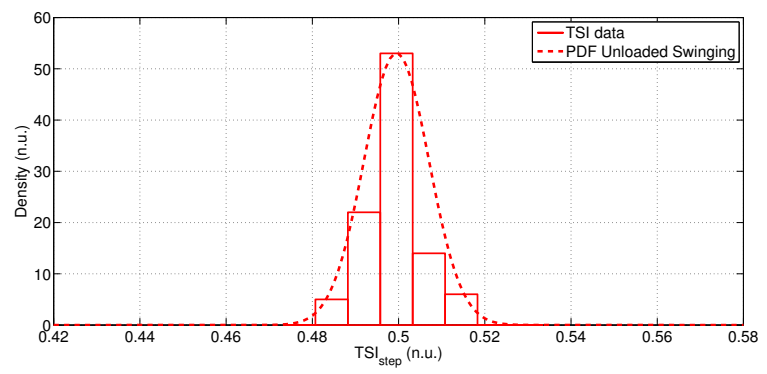

Fig. 7: Probability density function of the $\mathrm{TSI}_{\text {step }}$ for the unloaded swinging mode at $5 \mathrm{~km} / \mathrm{h}$ for one typical test participant

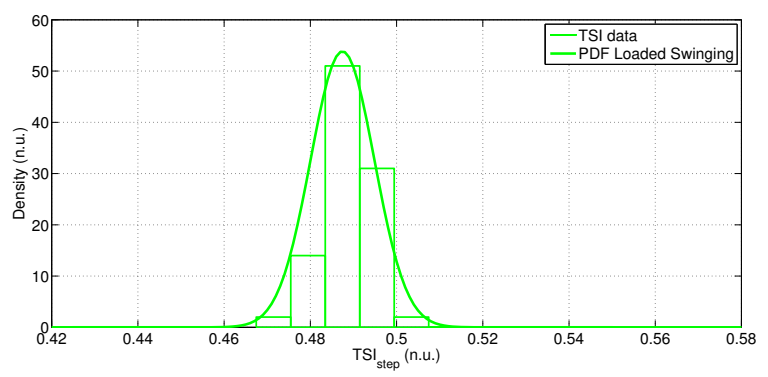

Fig. 8: Probability density function of the $\mathrm{TSI}_{\text {step }}$ for the loaded swinging mode at $5 \mathrm{~km} / \mathrm{h}$ for one test participant

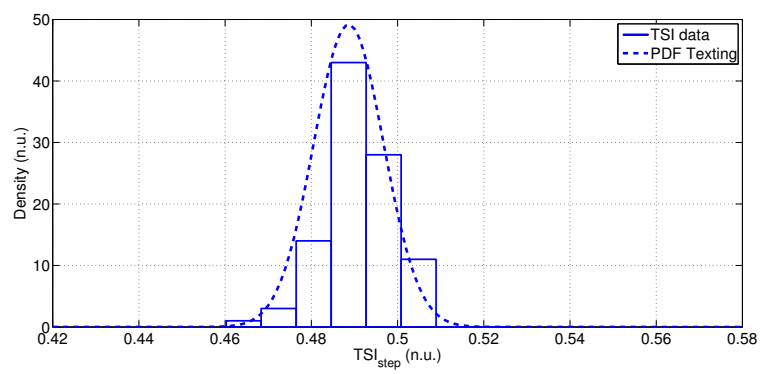

Fig. 9: Probability density function of the $\mathrm{TSI}_{\text {step }}$ for the texting mode at $5 \mathrm{~km} / \mathrm{h}$ for one test participant

The analysis of the walking gait symmetry over steps is conducted using the two-factor repeated measures analyze of variance (RM ANOVA) [32], [33]. To apply the ANOVA analysis, all TSIs are expected to follow Gaussian distributions for the different scenarios [32]. This is effectively observed with the experimental data sets and illustrated in Fig. 7, Fig.

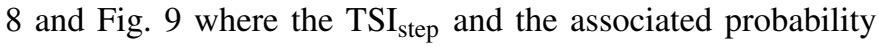
density functions are plotted for the three different scenarios at $5 \mathrm{~km} / \mathrm{h}$ for one test person. A shift of the Gaussian mean is observed on these three figures between the natural unloaded arm swinging case that is centered at 0.5 and the two other cases.
In total, 9000 strides were analyzed corresponding to the three carrying modes and the three walking speeds. RM ANOVA is applied to the $\mathrm{TSI}_{\text {step }}$ to assess possible changes of the walking gait symmetry over steps induced by the presence of a mass in hand. Two RM factors are considered. They are the device carrying mode factor and the walking speed factor. For the analysis, the three carrying modes and the three walking speeds are all processed together. The results of the RM ANOVA are given in Table I.

\section{TABLE I: RESULTS OF THE REPEATED MEASURES ANALYSIS (RM ANOVA) APPLIED TO TSI Ttep}

\begin{tabular}{l|ccccc} 
influencing factor & SS & df & MS & F & p \\
\hline Carrying mode (CM) & $9.3 \times 10^{-4}$ & 2 & $4.65 \times 10^{-4}$ & 5.530 & 0.013 \\
Walking speed (WS) & $8.8 \times 10^{-5}$ & 2 & $4.42 \times 10^{-5}$ & 0.243 & 0.7870 \\
CM x WS & $7.6 \times 10^{-4}$ & 4 & $1.90 \times 10^{-4}$ & 2.986 & 0.0316
\end{tabular}

In the RM ANOVA analysis, a F-test is used to find out whether the factors of interest impact the TSI $I_{\text {step }}$. The analysis is conducted using a variance ratio of two sets of $\mathrm{TSI}_{\text {step }}$ values. The derived $\mathrm{p}$ value must be below $5 \%$ to deduce that the studied factor has an impact on the TSI $I_{\text {step }}$ values. In Table $\mathrm{I}$, it is found that the carrying mode has a significant influence on the $\mathrm{TSI}_{\text {step }}$, and therefore the gait symmetry, with a $1.34 \%$ $\mathrm{p}$ value being below 5\%. On the contrary, the analysis shows that the walking speed factor has no significant influence on the gait symmetry since the corresponding $78.7 \% \mathrm{p}$ value is much greater than $5 \%$. The $\mathrm{p}$ value for the interaction of the factors "Carrying mode" and "Walking speed" equals 3.16\%, which is below 5\%. This result indicates that the influence of the carrying mode on the gait symmetry depends on the walking velocity. To deepen this analysis, the RM ANOVA is conducted on the TSI step for each walking speed $\left(V_{i}, i=1,2\right.$, 3 ) individually. The results are given in Table II.

\section{TABLE II: EFFECT OF CARRYING MODE FACTOR ON TSI $_{\text {step }}$ FOR THE DIFFERENT GAIT SPEEDS}

\begin{tabular}{l|ccc} 
& \multicolumn{3}{|c}{ Effect of carrying mode factor } \\
Walking speed & MS & F & p \\
\hline$V_{1}=3.6 \mathrm{~km} / \mathrm{h}$ & $5.1 \times 10^{-4}$ & 12.487 & $3.97 \times 10^{-4}$ \\
$V_{2}=5 \mathrm{~km} / \mathrm{h}$ & $1.71 \times 10^{-4}$ & 6.639 & $6.92 \times 10^{-3}$ \\
$V_{3}=5.8 \mathrm{~km} / \mathrm{h}$ & $1.66 \times 10^{-4}$ & 1.142 & 0.341
\end{tabular}

For higher walking speed, an increased $\mathrm{p}$ value is observed. The carrying mode has a significant influence on the gait symmetry at the step level for the lower speeds. Both $\mathrm{p}$ values for $V_{1}$ and $V_{2}$ are below 5\%. On the contrary, for the highest walking speed with a $34.1 \%$ p value, no significant effect of the carrying mode on gait step symmetry is observed.

Looking at each test participant, Fig. 10 shows the average $\mathrm{TSI}_{\text {step }}$ for all walking speeds with the three device carrying modes. All TSI numbers are expressed to four significant digits. A tendency may be concluded for the loaded swinging and texting cases with respect to unloaded swinging mode. Indeed, average $\mathrm{TSI}_{\text {step }}$ decreases for 8 participants out of 10 between the unloaded swinging mode and the two others. The average $\mathrm{TSI}_{\text {step }}$ mean value for all participants in the unloaded swinging mode is 0.4997. In loaded swinging and 


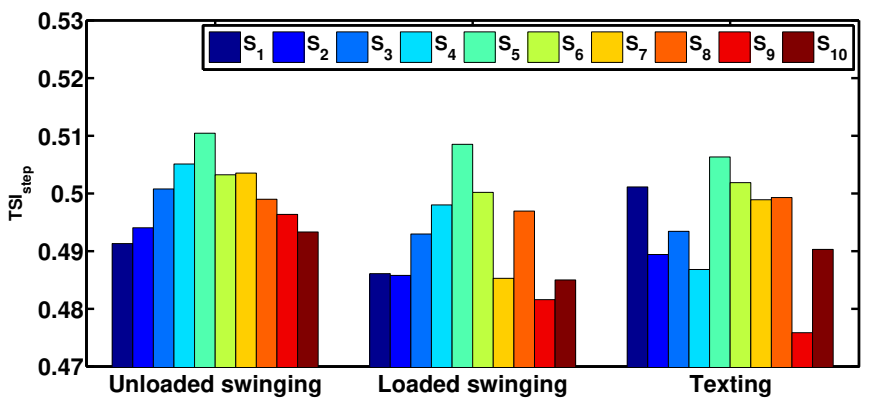

Fig. 10: Averaged TSI ${ }_{\text {step }}$ of each participant in the three device carrying modes (considering all the walking

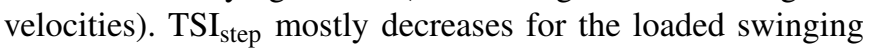
and texting cases with respect to unloaded swinging mode.

texting modes, a 0.4920 and 0.4943 average TSI $_{\text {step }}$ are found respectively.

To find out which device carrying mode has the greatest influence on the gait symmetry at the step level, the RM ANOVA analysis is conducted considering two combined factors: the unloaded swinging/loaded swinging and the unloaded swinging/texting. The $\mathrm{p}$ values are found to be equal to $0.13 \%$ and $8.35 \%$ respectively. We can conclude that the loaded swinging mode has a greater impact on the gait step symmetry than the texting mode. The same conclusion is found using a post-hoc analysis where the hypothesis $H_{0}$ stating that all average TSI are equal is tested.

Tukey's HSD (Honest Significant Difference) or Scheffé tests are classically used in conjunction with ANOVA analysis to determine where significant differences exist among more than two modalities. The Tukey's test gives a $7.1 \times 10^{-3} \mathrm{HSD}$ for our experimental data set. This value is a threshold that is compared to the separation between two means for assessing if the later are significantly different. Let us note $\mu_{1}, \mu_{2}$ and $\mu_{3}$ the mean values of respectively the unloaded swinging, loaded swinging and texting modes. The following three separations can be computed and compared to the HSD.

$$
\left\{\begin{array}{l}
\mu_{1}-\mu_{2}=7.67 \times 10^{-3}>7.1 \times 10^{-3} \\
\mu_{1}-\mu_{3}=5.38 \times 10^{-3}<7.1 \times 10^{-3} \\
\mu_{3}-\mu_{2}=2.29 \times 10^{-3}<7.1 \times 10^{-3}
\end{array}\right.
$$

These comparisons show that the unloaded swinging and loaded swinging modes are significantly different and none of these two modes is significantly different from the texting case. This is further illustrated in Fig. 11 where the texting and unloaded swinging modes, in blue and red respectively, overlap.

\section{IMPACT ON THE ESTIMATION OF TRAVELED DISTANCE FOR PEDESTRIAN NAVIGATION APPLICATIONS}

Existing step length models in pedestrian navigation algorithms usually assume that the walking gait is step/stride symmetric irrespective of the presence of a mass in hand. In the previous sections, it was shown that this is not the case. Because pedestrians can change directions at every step,

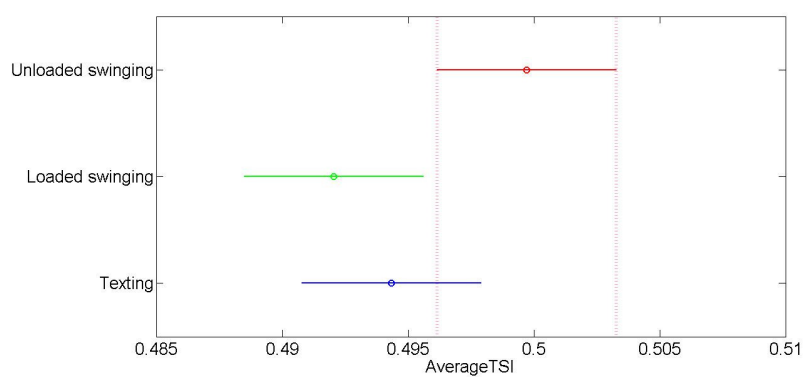

Fig. 11: Tuckey's HSD test for the three device carrying modes (considering all the walking velocities). Each group mean is represented by a small circle, and each comparison interval is represented by a bar extending out from the mean value. Two group means are significantly different if their intervals are disjoint. They are not significantly different if their intervals overlap.

it would not be sufficient to estimate only stride length. The risk would be to miss some angular changes in the walking direction and accumulate errors in the estimated PDR trajectories. Consequently it is interesting to assess the impact of this assumption on the traveled distance estimate.

\section{A. Position estimation error}

Let us quantify the position estimation error due to the walking gait asymmetry in a step symmetric modeling. An example of step length model is given by [13]. In this model, the step length $s$ is related to the step frequency $f$ and user's height $h$.Thus, if all steps are identical as assumed in PDR navigation algorithms, we have

$$
\Delta_{S_{D}}=\Delta_{S_{G}}
$$

where $\Delta_{S_{D}}$ is the right step duration and $\Delta_{S_{G}}$ is the left step duration. In this case, the stride duration is given by

$$
\Delta_{\text {Stride }}=\Delta_{S_{D}}+\Delta_{S_{G}}=2 \Delta_{S_{D}}
$$

Considering the asymmetry induced by the handheld device, Eq. 3 becomes

$$
\Delta_{S_{D}}=\text { TSI. } \Delta_{\text {Stride }}
$$

Thus, it is possible to estimate the induced error $\varepsilon$ and the error ratio $\varepsilon \%$ that are given by the following equations:

$$
\left\{\begin{array}{l}
\varepsilon=\left(2-\frac{1}{\text { TSI }}\right) \Delta_{S_{D}}=\left(\frac{2 \mathrm{TSI}-1}{\mathrm{TSI}}\right) \Delta_{S_{D}} \\
\varepsilon_{\%}=\left|\frac{2 \mathrm{TSI}-1}{\mathrm{TSI}}\right| \times 100
\end{array}\right.
$$

\section{B. Predicted errors based on empirical data}

In the experiments, $\mathrm{TSI}_{\text {step }}$ mean value is $\mu_{\mathrm{TSI}_{1}}=0.4920$ for the loaded swinging mode and $\mu_{\mathrm{TSI}_{2}}=0.4943$ for the texting mode, representing respectively the error ratios $\varepsilon_{1 \%}=3.25 \%$ and $\varepsilon_{2} \%=2.3 \%$. For a constant walking velocity, and considering $1 \mathrm{~m}$ long strides, the estimated error over a 100 $\mathrm{m}$ traveled distance without considering the gait asymmetry equals $3.25 \mathrm{~m}$ and $2.3 \mathrm{~m}$ for the loaded swinging and the texting modes respectively. These errors are significant for 
pedestrian navigation applications. Since the treadmill tends to make the gait more symmetric, higher errors are expected for overground walking. This suggests that the step length models should be improved for the context of handheld sensors.

TABLE III: Error ratio $\varepsilon_{\%}$ in $\%$ over the traveled distance for each participant and for the different trials

\begin{tabular}{l|rrrrrr} 
& \multicolumn{3}{|c}{ Loaded Swinging } & \multicolumn{3}{c}{ Texting } \\
participant & $\varepsilon \%\left(V_{1}\right)$ & $\varepsilon \%\left(V_{2}\right)$ & $\varepsilon \%\left(V_{3}\right)$ & $\varepsilon \%\left(V_{1}\right)$ & $\varepsilon \%\left(V_{2}\right)$ & $\varepsilon \%\left(V_{3}\right)$ \\
\hline $\mathrm{S}_{1}$ & 10.26 & 3.02 & 4.00 & 5.39 & 2.25 & 8.47 \\
$\mathrm{~S}_{2}$ & 14.94 & 0.82 & 2.34 & 6.78 & 5.72 & 0.56 \\
$\mathrm{~S}_{3}$ & 4.40 & 2.91 & 1.25 & 6.38 & 3.30 & 1.54 \\
$\mathrm{~S}_{4}$ & 0.07 & 1.14 & 3.66 & 0.19 & 3.53 & 13.38 \\
$\mathrm{~S}_{5}$ & 3.22 & 3.09 & 3.73 & 2.75 & 0.70 & 4.00 \\
$\mathrm{~S}_{6}$ & 2.54 & 2.22 & 0.50 & 0.26 & 0.58 & 1.92 \\
$\mathrm{~S}_{7}$ & 7.02 & 0.92 & 10.47 & 2.76 & 1.44 & 2.70 \\
$\mathrm{~S}_{8}$ & 6.02 & 0.93 & 1.23 & 0.89 & 0.26 & 0.21 \\
$\mathrm{~S}_{9}$ & 5.13 & 1.54 & 16.86 & 0.26 & 9.78 & 22.09 \\
$\mathrm{~S}_{10}$ & 9.57 & 4.10 & 4.95 & 0.86 & 8.61 & 2.53 \\
\hline Mean & 6.32 & 2.07 & 4.90 & 2.65 & 3.61 & 5.74
\end{tabular}

In Table III, the position estimation error ratio $\varepsilon \%$ corresponding to each trial (carrying mode / walking speed) is reported for all test participants. These values correspond to the average $\mathrm{TSI}_{\text {step }}$ values. In the loaded swinging mode, the impact on traveled distance estimation is found to be greater at lower speed $\left(\varepsilon \%\left(V_{1}\right)=6.32 \%\right)$ than at higher walking velocities. Indeed, at higher speed, the synchronization between the arms and the legs increases and the impact of the added mass is found to be less significant. On the contrary, for the texting mode, the fact of walking with rigid upper limbs has a greater impact on the position estimation at higher walking velocities $\left(\varepsilon_{\%}\left(V_{3}\right)=5.74 \%\right)$. In this scenario, the loaded arm is not synchronized with the legs movement. This outcome completes the results found in table II.

\section{Comparison with predicted errors from simulation}

Based on simulation model, The position estimation error ratio is calculated for the walking velocities $V_{1}, V_{2}$ and $V_{3}$ to compare with the results based on the empirical data. The error ratios corresponding to loaded swinging and texting modes are shown in table IV.

TABLE IV: Error ratio $\varepsilon \%$ in $\%$ based on simulation results for all testing speeds and for different carrying modes

\begin{tabular}{l|rrrrrr}
\multirow{2}{*}{ Gait speed } & \multicolumn{3}{|c}{ Loaded Swinging } & \multicolumn{4}{c}{ Texting } \\
& $V_{1}$ & $V_{2}$ & $V_{3}$ & $V_{1}$ & $V_{2}$ & $V_{3}$ \\
\hline$\varepsilon_{\%}$ & 1.94 & 1.38 & 1.87 & 1.08 & 2.52 & 3.04
\end{tabular}

The error ratios calculated based on the conceived human gait model are much lower than mean values found with experimentation data. This is due to several limitations of the proposed model. The identified limitations and recommendations for future work are the following:

- It has been assumed that most features of human walking can be captured by analyzing it in sagittal plane. This is true for leg movements. However, the observation of the positions of the hand markers shows that humans tend to increase their movement in the horizontal plane with an increase in walking speed. Hence, to study human walking with arm swinging, 3D model should be considered.

- For the simplicity of walking gait cycle design, DS phases were neglected. Humans have a well-defined DS phase which should not be neglected to capture all the features of human walking gait in the simulation.

- The simulation tool is unable to model the cognitive effects of interacting with a device (dual-task paradigm), interaction with other individuals, and making maneuvers.

\section{CONCLUSION}

Using a human gait simulation over a stride with upper extremity model, it was found that the presence of a mass in hand, corresponding for example to a smartphone, alters the step symmetry of the walking gait. This outcome, which is further investigated with experiments in this paper, is of main interest for health monitoring applications based on connected objects.

The impact of the handheld mass on the walking gait is studied with experimental data collected with 10 test participants and a $0.19 \mathrm{~kg}$ handheld device in a motion capture laboratory. It is shown that the presence of a mass in hand changes the gait symmetry at the step level whereas the gait remains stride symmetric. As compared to a naturalistic walking (i.e. without any mass in hand), it is also found that both carrying modes: mass held in a swinging hand and in a "texting" hand, which corresponds to a rigid arm with the eyes of the person looking at the object's screen, have impact on the walking gait. Finally, for swinging mode, the impact of carrying a mass in hand is found to be greater at lower speeds $(3.6 \mathrm{~km} / \mathrm{h})$ than at comfortable/faster speeds $(5-5.8 \mathrm{~km} / \mathrm{h})$ while an inverse tendency is observed for texting mode. These findings tend to prove the importance of natural arm swinging that could be captured with handheld devices.

Another important result for the field of pedestrian navigation based on inertial navigation systems is that the presence of a handheld mass should be considered to adapt step length models that are used in PDR processing strategy. Indeed, it is commonly assumed in the literature that all the steps are identical when a human is walking with a handheld device. The outcome of this research shows that this assumption is a source of error in the computation of the cumulative traveled distance. In the long term, this study attempts to solve for the direct modeling problem of human displacements solely based data from a handheld inertial mobile unit classically embedded in the connected object. The goal is to deduce the position of the center of mass of the human using inertial and satellites data recorded by the handheld device.

Finally this research shows that combining the two research areas, i.e. biomechanics and pedestrian navigation, is a very interesting way of addressing the challenges related to the increase of individual's autonomy using everyday objects.

\section{REFERENCES}

[1] L. Atallah, B. Lo, R. King, and G. Yang, "Sensor positioning for activity recognition using wearable accelerometers," IEEE Trans. Biomed. Circuits Syst., vol. 5, no. 4, pp. 320-329, Aug. 2011. 
[2] J. Margarito, R. Helaoui, A. M. Bianchi, F. Sartor, and A. G. Bonomi, "User-Independent Recognition of Sports Activities from a Single Wristworn Accelerometer: A Template Matching Based Approach," IEEE Trans. Biomed. Eng., vol. 63, no. 4, pp. 788-796, Apr. 2016.

[3] M. Susi, V. Renaudin, and G. Lachapelle, "Motion mode recognition and step detection algorithms for mobile phone users," Sensors, vol. 13, pp. 1539-1562, 2013.

[4] I. C. Gyllensten and A. G. Bonomi, "Identifying types of physical activity with a single accelerometer: Evaluating laboratory-trained algorithms in daily life," IEEE Trans. Biomed. Eng., vol. 58, no. 9, pp. 2656-2663, Sep. 2011.

[5] E. D. Kaplan and C. Hegarty, Understanding GPS Principles and Applications, Artech House, 2nd Ed., 2006.

[6] N. E. Klepeis et al., "The National Human Activity Pattern Survey (NHAPS): a resource for assessing exposure to environmental pollutants," J. Expo. Anal. Environ. Epidemiol., vol. 11, no. 3, pp. 231-252, 2001.

[7] C. Combettes and V. Renaudin, "Delay Kalman Filter to Estimate the Attitude of a Mobile Object with Indoor Magnetic Field Gradients," Micromachines., vol. 7, no. 5, pp. 17p, Jan. 2016.

[8] D. H. Titterton and J. L. Weston, Strapdown Inertial Navigation Technology, 2nd Ed. Institution of Electrical Engineers, Stevenage, 2004.

[9] P. D. Groves, G. W. Pulford, C. A. Littlefield, D. L. J. Nash, and C. J. Mather, "Inertial Navigation Versus Pedestrian Dead Reckoning: Optimizing the Integration," in 20th International Technical Meeting of the Satellite Division of The Institute of Navigation, 2007, pp. 2043-2055.

[10] I. Skog, P. Händel, J. Nilson, and J. Rantakokko, "Zero-Velocity Detection - An Algorithm Evaluation," IEEE Trans. Biomed. Eng., vol. 57, no. 11, pp. 2657-2666, 2010.

[11] S. F. Donker, P. J. Beek, R. C. Wagenaar, and T. Mulder, "Coordination Between Arm and Leg Movements During Locomotion," J. Mot. Behav., vol. 33, no. 1, pp. 86-102, 2001.

[12] S. H. Collins, P. G. Adamczyk, and A. D. Kuo, "Dynamic arm swinging in human walking," Proc. R. Soc. B, vol. 276, pp. 3679-3688, Jul. 2009.

[13] V. Renaudin, M. Susi, and G. Lachapelle, "Step Length Estimation Using Handheld Inertial Sensors," Sensors, vol. 12, no. 7, pp. 8507-8525, Jun. 2012.

[14] J. Jahn, U. Batzer, J. Seitz, L. Patino-Studencka, and J. G. Boronat, "Comparison and evaluation of acceleration based step length estimators for handheld devices," in International Conference on Indoor Positioning and Indoor Navigation, Zurich, Switzerland, 2010, pp. 1-6.

[15] M. L. McGuire, "An Overview of Gait Analysis and Step Detection in Mobile Computing Devices," in 4th International Conference on Intelligent Networking and Collaborative Systems, Bucharest, Romania, 2012, pp. 648-651.

[16] S. Ha, J. Yu, Y. Han, and H. Hahn, "Adaptive Gait Pattern Generation of Biped Robot based on Human's Gait Pattern Analysis," World Acad Sci Eng Technol., Vol. 23, pp. 406-411, Aug. 2007.

[17] G. Capi and M. Yokota, "Optimal Multi -criteria humanoid robot gait synthesis-an evolutionary approach," Int J Innov Comput I, vol. 2, no. 6, pp. 1249-1258, Dec. 2006

[18] M. Y. Cheng and C. S. Lin, "Genetic Algorithm for Control Design of Biped Locomotion," J Robotic Syst, Vol. 14, no. 5, pp. 365-373, 1997.

[19] Y. Hasagawa, T. Arakawa, and T. Fukuda, "Trajectory generation for biped locomotion robot," Journal Mechatronics, Vol. 10, pp. 67-89, 2000.

[20] J. H. Park and M. Choi, "Generation of an Optimal Gait Trajectory for Biped Robots Using a Genetic Algorithm," JSME International Journal, Vol. 47, No. 2, pp. 715-721, 2004.

[21] B. I. Prilutsky and V. M. Zatsiorsky, "Optimization-based models of muscle coordination," Exercise and Sport Sciences Reviews, vol. 1, pp. $32-38,2002$.

[22] B. Kaddar, Y. Aoustin, and C. Chevallereau, "Arm swing effects on walking bipedal gaits composed of impact, single and double support phases," Robot. Auton. syst., vol. 66, pp. 104-115, Apr. 2015.

[23] B. Kaddar, "Effet du balancement des bras sur la consommation énergétique durant la marche d'un robot bipède," Ph.D. thesis (in French), Nantes University, France, 2013.

[24] R. Dumas, L. Chèze, and J. P. Verriest, "Adjustments to Mcconville et al. and Young et al. body segment inertial parameters," J. Biomech., vol. 40, pp. 543-553, 2007.

[25] P. De Leva, "Adjustments to zatsiorskyseluyanov's segment inertia parameters," J. Biomech., vol. 29, no. 9, pp. 1223-1230, 1996.

[26] S. Kumar, V. Renaudin, Y. Aoustin, E. Le-Carpentier, and C. Combettes, "Model-based and experimental analysis of the symmetry in human walking in different device carrying modes," in 6th IEEE International Conference on Biomedical Robotics and Biomechatronics (BioRob), Singapore, Jun. 2016, pp. 1172-1179.
[27] M. J. P. Powell, Variable Metric Methods for Constrained Optimization. Lecture Notes in Mathematics, pp. 62-72. Springer, Berlin (1977)

[28] C. Chevallereau and Y. Aoustin, "Optimal reference trajectories for walking and running of a biped," Robotica, vol. 19, no. 5, pp. 557-569, 2001.

[29] J. Perry and J. M. Burnfield, Gait analysis: Normal and pathological function, 2nd Ed. pp. 121-130. Pomona, CA, USA: Slack Incorporated, Feb. 2010.

[30] M. L. Harris-Love, L. W. Forrester, R. F. Macko, K. H. Silver, and G. V. Smith, "Hemiparetic gait parameters in overground versus treadmill walking "Neurorehabilitation and Neural Repair, Vol. 15, no. 2, pp. 105-112, Mar. 2001.

[31] J. Rose and J. G. Gamble, Human walking, 3rd Ed. Philadelphia, PA, USA: Lippincott Williams \& Wilkins, 2006.

[32] P. J. Potvin and R. W. Schutz, "Statistical power for the two-factor repeated measures ANOVA," Behav. Res. Meth. Ins. C., vol. 32, no. 2, pp. 347-356, 2000

[33] Analyse de la variance ANOVA. http://math.univlyon1.fr/ duheille/MASS42_anova.pdf. Accessed 20 December 2015

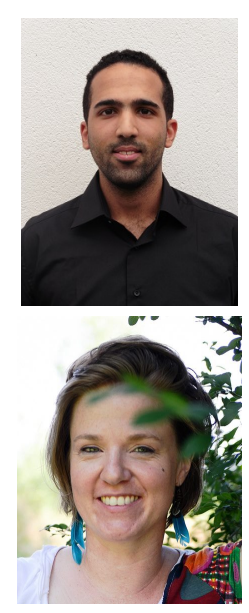

Mahdi Abid received the M.S. degree in robotics and automatics control from Montpellier 2 University, Montpellier, France, in 2015. He is currently a Ph.D. student in the Geopositioning Laboratory at IFSTTAR, Bouguenais, France. His research interests include humanoid robotics, human motion generation, human gait analysis, and system identification.

Valérie Renaudin received the M.Sc. degree in geomatics engineering from ESGT, France and the $\mathrm{Ph} . \mathrm{D}$. degree in computer, communication, and information sciences from EPFL, Switzerland. She was a Technical Director with Swissat Company, developing real-time geopositioning solutions based on a permanent GNSS network and a Senior Research Associate with PLAN Group, University of Calgary, Canada. She is currently leading the Geopositioning Laboratory at IFSTTAR. Her research focuses on outdoor/indoor navigation using GNSS, inertial and magnetic data, particularly for pedestrians to improving sustainable personal mobility. She is the recipient of the European Marie Curie Career Integration Grant for the smartWALK project.

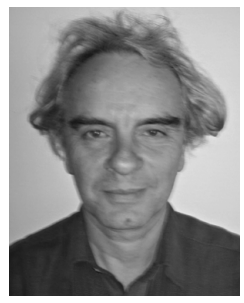

Yannick Aoustin received the Ph.D. degree in Automatic in 1987 and his research degree for leading research of Ph.D. students in 2006, both from the University of Nantes, Nantes, France. He is currently a Lecturer Excluding class at the Department of Physics, University of Nantes, Nantes, France. His research interests include mechanical systems under actuated legged robots, bipedal, nonlinear observers, and biomechanics, He has many publications on journals circulated internationally and premier conferences. He is also an Associate Editor of International Journal of Advanced Robotic Systems.

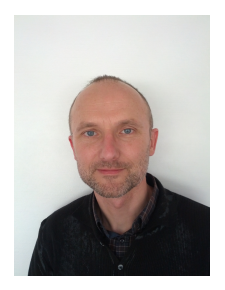

Eric Le Carpentier received the Ph.D. degree in automatic control from the Nantes University, Nantes, France, in 1990.

Since 1992, he has been an Assistant Professor at Ecole Centrale de Nantes, France, and at the Laboratoire des Sciences du Numérique de Nantes. His current research interests include signal processing, stochastic simulation for estimation, detection, tracking and control, with applications in biomedical signals, robotics and precise geo-location.

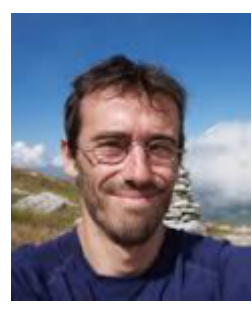

Thomas Robert received his M.S. degree in Mechanical Engineering in 2002 and his Ph.D. in biomechanics in 2006, both from the National Institute of Applied Science in Lyon, France.

Since 2008, he has been a Researcher in the Biomechanics and Impact Mechanics Laboratory (IFSTTAR - Université Lyon 1) in Lyon. His main research interests are the analysis of human movement dynamics and the balance of human in dynamic situations. 